\title{
A POLYNOMIAL ALGORITHM FOR MINDSC ON A SUBCLASS OF SERIES PARALLEL GRAPHS
}

\author{
Salim Achouri ${ }^{1}$, Timothée Bossart ${ }^{1}$ \\ And Alix Munier-Kordon ${ }^{1}$
}

\begin{abstract}
The aim of this paper is to show a polynomial algorithm for the problem minimum directed sumcut for a class of series parallel digraphs. The method uses the recursive structure of parallel compositions in order to define a dominating set of orders. Then, the optimal order is easily reached by minimizing the directed sumcut. It is also shown that this approach cannot be applied in two more general classes of series parallel digraphs.
\end{abstract}

Keywords. Minimum directed sumcut, series parallel graph, polynomial algorithm.

Mathematics Subject Classification. 05C78, 05C85, 90 B35.

\section{INTRODUCTION}

Graph ordering problems under a cost criterion lead to many applications in computer science (e.g. the minimization of the required number of registers for the execution of a program on a single machine [6]). Let $G=(V, A)$ be a digraph, a bijection $\varphi \mapsto\{1,2, \ldots,|V|\}$ is called an order of $G$ if $\forall(u, v) \in A, \varphi(u)<\varphi(v)$.

The scope of this paper is the criterion directed sumcut (DSC), which is a generalization to directed graphs of the criterion sumcut [7]. It was proven in [1] that DSC models the sum of variable lifespans for a program. It is defined in the following way: for any order $\varphi$, the cost of a vertex $u \in V$ is

$$
\mathcal{C}(\varphi, u)=\max _{v \in \Gamma^{+}(u)} \varphi(v)-\varphi(u),
$$

Received June 30, 2007. Accepted July 3, 2008.

1 LIP6 - Université Pierre et Marie Curie, 4 place Jussieu, 75252 Paris Cedex 05, France;

Alix.Munier@lip6.fr 
where $\Gamma^{+}(u)$ is the set of immediate successors of $u$. The cost of $\varphi$ for $G$ is

$$
\mathcal{C}(\varphi, G)=\sum_{u \in V} \mathcal{C}(\varphi, u)
$$

Let $V^{\prime} \subset V$, and let $G^{\prime}=\left(V^{\prime}, A^{\prime}\right)$ be a partial subgraph of $G$. The cost of $\varphi$ for $G^{\prime}$ is simply

$$
\mathcal{C}\left(\varphi, G^{\prime}\right)=\sum_{u \in V^{\prime}} \mathcal{C}(\varphi, u)
$$

The directed sum cut (DSC) of $G$ is the minimum value $\mathcal{C}(G)=\min _{\varphi} \mathcal{C}(\varphi, G)$. minDSC was proven to be NP-complete for bipartite graphs [2] and polynomial for intrees and outtrees in [1]. The aim of this paper is to show a polynomial algorithm for another recursively-described graph class, the series parallel graphs. The most common definitions of this class are 2TSPG (2-terminal series parallel graphs) $[5,8]$ and SP [3]. However, we show in Section 4 that the recursive structure does not yield a dominating set of orders for both classes. The class we are interested in this paper is a subclass of both, denoted r-2TSPG (for reduced 2-terminal series parallel graphs). The main interest of r-2TSPG is the limitation of the number of arcs between a component and the corresponding upper-level component. This caracteristic allows us to simplify the criterion evaluation. This class was previously introduced in [4] for a scheduling problem with communication delays.

Definition 1.1 (r-2TSPG graph). The graph consisting of 2 vertices $s$ and $t$ connected by a single arc $(s, t)$ is the basic series parallel graph for r-2TSPG. Compound graphs can be obtained from $K$ smaller ones $G_{i}, 1 \leq i \leq K$, of respective source and sink $s_{i}$ and $t_{i}$ according to two composition rules:

Series: Identify $p_{i}$ with $t_{i+1}, \forall i, 1 \leq i \leq K-1$. The source and sink of $G$ are respectively $s_{1}$ and $t_{K}$.

Parallel: Create the source and sink of $G s$ and $t$. Create the arcs $\left(s, s_{i}\right)$ and $\left(t_{i}, t\right) \forall i, 1 \leq i \leq K$.

In Section 2, we show the dominance of block orders for DSC on r-2TSPG. In Section 3, we prove that an optimal order can be reached in polynomial time. Finally, we conclude (Sect. 4) that the algorithm does not apply in 2TSPG and in $\mathrm{SP}$, with simple counter examples.

\section{DominANCE OF BLOCK ORDERS}

Let $G=(V, A) \in \mathrm{r}-2 \mathrm{TSPG}$ be the parallel composition of $G_{i}=\left(V_{i}, A_{i}\right), 1 \leq$ $i \leq K$. Let $s$ and $t$ denote the source and sink vertices of $G$, and let $s_{i}$ and $t_{i}$ be the respective source and sink vertices of $G_{i}$. An example can found in Figure 1.

Let $\varphi=\varphi^{(0)}$ be an order of $G$. For $i \in\{1, \ldots, K\}$ and $j \in\left\{1, \ldots, k_{i}\right\}$, we denote $B_{i}^{j}$ the $j$ th maximal sequence of consecutive vertices of $G_{i}$ w.r.t. $\varphi$. $k_{i}$ is thus the number of such $B_{i}^{j}$ 's. For the example pictured by Figure 2, we obtain $k_{1}=3$ and $k_{2}=k_{3}=2$. 


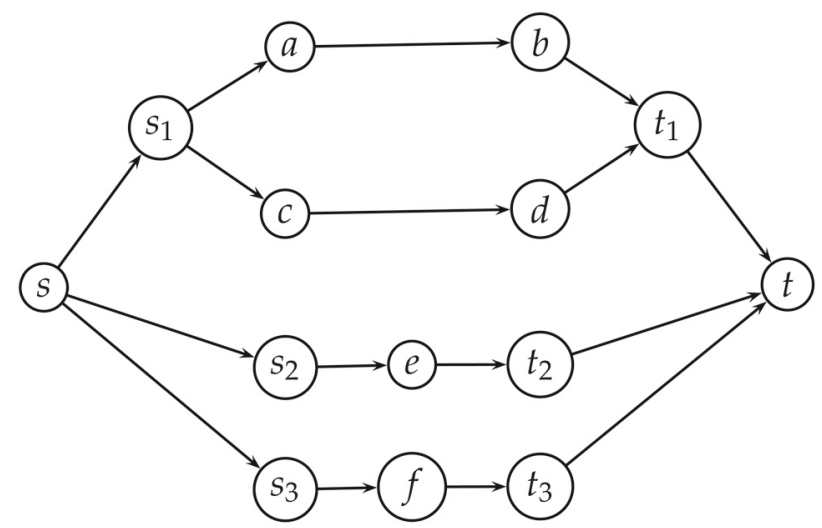

Figure 1. A graph $G=(V, A)$ corresponding to a parallel composition of the subgraphs $G_{1}=\left(\left\{s_{1}, a, b, c, d, t_{1}\right\}, A\right), G_{2}=$ $\left(\left\{s_{2}, e, t_{2}\right\}, A\right)$ and $G_{3}=\left(\left\{s_{3}, f, t_{3}\right\}, A\right)$.
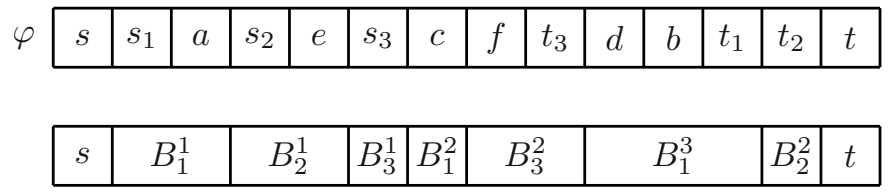

FiguRE 2. An order $\varphi$ for the graph pictured by Figure 1 and the corresponding blocks.

Lastly, $\Omega_{\varphi}$ denotes the set of arcs which actually bear a cost in terms of the DSC criterion:

$$
\Omega_{\varphi}=\left\{(z, w) \in G, \varphi(w)=\max _{v \in \Gamma^{+}(z)} \varphi(v)\right\} .
$$

For all $z \in V-\{t\}$, the vertex $v \in V$ such that $(z, v) \in \Omega_{\varphi}$ is called the last successor of $z$.

Definition 2.1 (Block order). $\varphi$ is a block order if $\forall i \in\{1, \ldots, K\}, \forall(x, y) \in$ $V_{i} \times V_{i}, \forall z \in V$ such that $\varphi(x)<\varphi(z)<\varphi(y)$, then $z \in V_{i}$.

For the example pictured by Figures 1 and 2, we get

$$
\Omega_{\varphi}=V \backslash\left\{\left(s, s_{1}\right),\left(s, s_{2}\right),\left(s_{1}, a\right)\right\} .
$$

When $\varphi$ is a block order, we can also consider an order called block labelling function, which consists in numbering blocks (see Sect. 3 for a more formal definition). Throughout this paper, we only consider parallel compositions, as series compositions are trivial in terms of DSC cost since all vertices from $G_{i}$, 


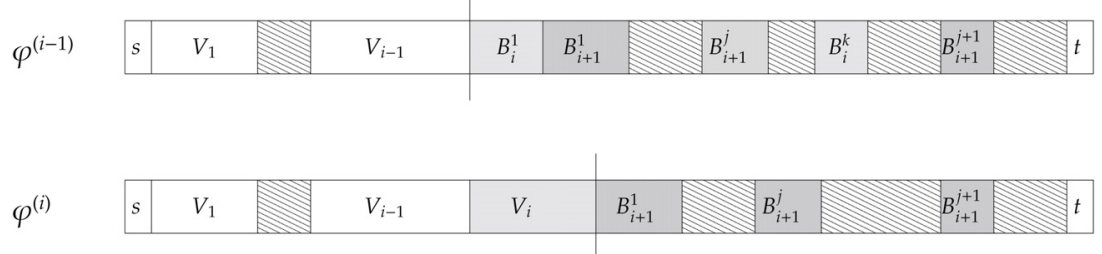

Figure 3. Reordering $\varphi^{(i-1)}$ to $\varphi^{(i)}$.

$i \in\{1, \ldots, K-1\}$ must be ordered before vertices from $G_{i+1}$. We show in this section that for any order, it is always possible to build a block order with no greater cost. We build a block order by successive concatenations of vertex sets belonging to the same subgraph. Step $i, i \in\{1, \ldots, K-1\}$ consists in the concatenation of sets $B_{i}^{j}, j \in\left\{1, \ldots, k_{i}\right\}$ with $B_{i}^{1}, i$ being the lowest index of not-yet-concatenated subgraphs (see Fig. 3). For every $i_{0} \in\{1, \ldots, K\}$ such that $k_{i_{0}}=1$, we simply consider that $\varphi^{\left(i_{0}\right)}=\varphi^{\left(i_{0}-1\right)}$. Therefore, we only consider in the following steps $i$ verifying $k_{i}>1$.

Clearly, $\varphi^{(K-1)}$ is a block order. We show in the following that for every $i \in\{1, \ldots, K-1\}$,

$$
\mathcal{C}\left(\varphi^{(i)}, G\right)-\mathcal{C}\left(\varphi^{(i-1)}, G\right) \leq 0 .
$$

Since $\mathcal{C}\left(\varphi^{(i)}, G_{l}\right)=\mathcal{C}\left(\varphi^{(i-1)}, G_{l}\right), \forall l \in\{1, \ldots, i-1\}$, we can write

$$
\begin{aligned}
\mathcal{C}\left(\varphi^{(i)}, G\right)-\mathcal{C}\left(\varphi^{(i-1)}, G\right)= & \mathcal{C}\left(\varphi^{(i)}, s\right)-\mathcal{C}\left(\varphi^{(i-1)}, s\right)+\mathcal{C}\left(\varphi^{(i)}, G_{i}\right)-\mathcal{C}\left(\varphi^{(i-1)}, G_{i}\right) \\
& +\mathcal{C}\left(\varphi^{(i)}, G_{i+1}\right)-\mathcal{C}\left(\varphi^{(i-1)}, G_{i+1}\right) \\
& +\sum_{l=i+2}^{K} \mathcal{C}\left(\varphi^{(i)}, G_{l}\right)-\mathcal{C}\left(\varphi^{(i-1)}, G_{l}\right) .
\end{aligned}
$$

Lemma 2.2. $\forall i \in\{1, \ldots, K-1\}, \mathcal{C}\left(\varphi^{(i)}, s\right)-\mathcal{C}\left(\varphi^{(i-1)}, s\right) \leq \varphi^{(i)}\left(s_{i+1}\right)-\varphi^{(i-1)}\left(s_{i+1}\right)$.

Proof. The last successor of $s$ in $\varphi^{(i)}$ and $\varphi^{(i-1)}$ is $s_{K}$. Therefore,

$$
\mathcal{C}\left(\varphi^{(i)}, s\right)-\mathcal{C}\left(\varphi^{(i-1)}, s\right)=\varphi^{(i)}\left(s_{K}\right)-\varphi^{(i-1)}\left(s_{K}\right) .
$$

From $\varphi^{(i-1)}$ to $\varphi^{(i)}$, the number of vertices of $V$ renumbered between $s_{i}$ and $s_{K}$ is at most $\left|V_{i}\right|-\left|B_{i}^{1}\right|$. Therefore,

$$
\left|V_{i}\right|-\left|B_{i}^{1}\right| \geq \varphi^{(i)}\left(s_{K}\right)-\varphi^{(i-1)}\left(s_{K}\right) .
$$

Now, $\varphi^{(i)}\left(s_{i+1}\right)=\varphi^{(i)}\left(s_{i}\right)+\left|V_{i}\right|$ and $\varphi^{(i-1)}\left(s_{i+1}\right)=\varphi^{(i-1)}\left(s_{i}\right)+\left|B_{i}^{1}\right|=\varphi^{(i)}\left(s_{i}\right)+$ $\left|B_{i}^{1}\right|$. By adding both equations we have $\left|V_{i}\right|-\left|B_{i}^{1}\right|=\varphi^{(i)}\left(s_{i+1}\right)-\varphi^{(i-1)}\left(s_{i+1}\right)$, hence the result. 


\begin{tabular}{|c|c|c|c|}
\hline$j$ & 1 & 2 & 3 \\
\hline$\Theta_{1}^{j}$ & $\left\{s_{1}, a\right\}$ & $\{c\}$ & $\left\{t_{1}\right\}$ \\
\hline$\Theta_{2}^{j}$ & $\{e\}$ & $\left\{t_{2}\right\}$ & undefined \\
\hline$\Theta_{3}^{j}$ & $\left\{s_{3}\right\}$ & $\left\{t_{3}\right\}$ & undefined \\
\hline
\end{tabular}

TABLE 1. Sets $\Theta_{i}^{j}$ with $i \in\{1, \ldots, K\}$ and $j \in\left\{1, \ldots, k_{i}\right\}$ for the example pictured by Figures 1 and 2 .

Lemma 2.3. $\forall i \in\{1, \ldots, K-1\}, \quad \sum_{l=i+2}^{K}\left(\mathcal{C}\left(\varphi^{(i)}, G_{l}\right)-\mathcal{C}\left(\varphi^{(i-1)}, G_{l}\right)\right) \leq 0$.

Proof. Let $(v, w) \in \Omega_{\varphi^{(i)}}$ with $(v, w) \in V_{l} \times\left(V_{l} \cup\{t\}\right), l \in\{i+2, \ldots, K\}$.

(a) If there exists $\beta \in\left\{1, \ldots, k_{l}\right\}$ such that $v$ and $w$ belong to a same subset $B_{l}^{\beta}$, then

$$
\begin{aligned}
\mathcal{C}\left(\varphi^{(i-1)}, v\right) & =\varphi^{(i-1)}(w)-\varphi^{(i-1)}(v) \\
& =\varphi^{(i)}(w)-\varphi^{(i)}(v) \\
& =\mathcal{C}\left(\varphi^{(i)}, v\right) .
\end{aligned}
$$

(b) Otherwise, let $\beta_{1}, \beta_{2} \in\left\{1, \ldots, k_{l}\right\}^{2}$ with $v \in B_{l}^{\beta_{1}}$ and $w \in B_{l}^{\beta_{2}}$. Then, all vertices from subsets $B_{i}^{j}$ numbered by $\varphi^{(i-1)}$ between $B_{l}^{\beta_{1}}$ and $B_{l}^{\beta_{2}}$ are numbered by $\varphi^{(i)}$ before $B_{l}^{1}$. Therefore,

$\mathcal{C}\left(\varphi^{(i-1)}, v\right)=\varphi^{(i-1)}(w)-\varphi^{(i-1)}(v) \geq \varphi^{(i)}(w)-\varphi^{(i)}(v)=\mathcal{C}\left(\varphi^{(i)}, v\right)$.

We deduce that for every $l \in\{i+2, \ldots, K\}$,

$$
\mathcal{C}\left(\varphi^{(i)}, G_{l}\right)-\mathcal{C}\left(\varphi^{(i-1)}, G_{l}\right) \leq 0 .
$$

We define for every $i \in\{1, \ldots, K\}$ and for every $j \in\left\{1, \ldots, k_{i}-1\right\}$,

$$
\Theta_{i}^{j}(\varphi)=\left\{z,(z, v) \in \Omega_{\varphi}, z \in B_{i}^{j} \text { and } v \in \bigcup_{l=j+1}^{k_{i}} B_{i}^{l}\right\} .
$$

We also define $\Theta_{i}^{k_{i}}(\varphi)=\left\{t_{i}\right\}=\left\{z,(z, t) \in \Omega_{\varphi}, z \in B_{i}^{k_{i}}\right\}$. Finally, for every $i \in\{1, \ldots, K\}$ and for every $j \in\left\{1, \ldots, k_{i}\right\}$, we set $\theta_{i}^{j}(\varphi)=\left|\Theta_{i}^{j}(\varphi)\right|$. Sets $\Theta_{i}^{j}$ corresponding to our example pictured by Figures 1 and 2 are given by Table 1 .

Lemma 2.4. Let $\varphi$ be an order of $G$. For every $i \in\{1, \ldots, K\}$ and for every $j \in\left\{1, \ldots, k_{i}\right\}, \theta_{i}^{j}(\varphi)>0$.

Proof.

(a) $\theta_{i}^{k_{i}}(\varphi)=\left|\Theta_{i}^{k_{i}}(\varphi)\right|=1$. 


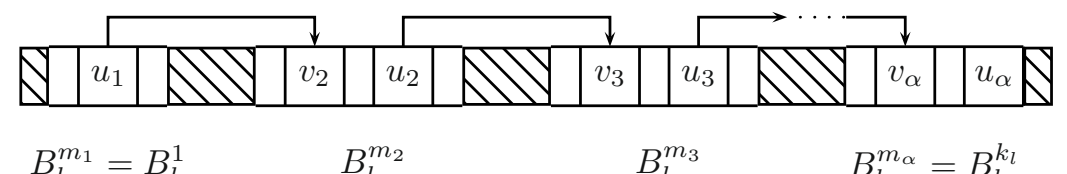

$$
B_{l}^{m_{1}}=B_{l}^{1} \quad B_{l}^{m_{2}} \quad B_{l}^{m_{3}} \quad B_{l}^{m_{\alpha}}=B_{l}^{k_{l}}
$$

FigURE 4. Sequences $\left(u_{\beta}\right)_{\beta \in\{1, \cdots, \alpha\}}$ and $\left(v_{\beta}\right)_{\beta \in\{2, \cdots, \alpha\}}$.

(b) For any $j \in\left\{1, \ldots, k_{i}-1\right\}$, let $u$ be the last element from $B_{i}^{j}$ following $\varphi$. There exists a path in $G$ from $u$ to $t_{i}$, so $\Gamma^{+}(u) \neq \emptyset$. Moreover, $\Gamma^{+}(u) \subseteq \bigcup_{l=j+1}^{k_{i}} B_{i}^{l}$. Then, there exists $v \in \bigcup_{l=j+1}^{k_{i}} B_{i}^{l}$ with $(u, v) \in \Gamma_{\varphi}$, so $u \in \Theta_{i}^{j}(\varphi)$ and $\theta_{i}^{j}(\varphi)>0$.

Particular paths from $G$ will be pointed up in the following lemmas. For a couple of fixed values $i \in\{1, \ldots, K-1\}$ and $l \in\{i, \ldots, K\}$, a strictly increasing sequence of $\alpha>0$ integers $m_{\beta} \in\left\{1, \ldots k_{l}\right\}$ and two sequences of vertices $u_{\beta}, \beta \in\{1, \ldots, \alpha\}$ and $v_{\beta}, \beta \in\{2, \ldots, \alpha\}$ are defined as follows:

(1) $m_{1}=1, m_{\alpha}=k_{l}$;

(2) for every $\beta \in\{1, \ldots, \alpha-1\},\left(u_{\beta}, v_{\beta+1}\right) \in \Omega_{\varphi^{(i-1)}}$ with $u_{\beta} \in B_{l}^{m_{\beta}}$ and $v_{\beta+1} \in B_{l}^{m_{\beta+1}}$

(3) $u_{\alpha}=t_{l}$.

Figure 4 illustrates the definition of these 3 sequences. By Lemma $2.4, \theta_{l}^{k}\left(\varphi^{(i-1)}\right)>$ 0 for every $k \in\left\{1, \ldots, k_{l}\right\}$, so these sequences exist.

For the example pictured by Figures 1 and 2 and fixed values $i=l=1$, a sequence with $\alpha=3$ terms can be defined as $m_{1}=1, m_{2}=2$ and $m_{3}=3$ with $u_{1}=s_{1}, u_{2}=c, u_{3}=t_{1}, v_{2}=c$ and $v_{3}=d$.

For every $i \in\{1, \ldots, K-1\}$ and $j \in\left\{1, \ldots, k_{i}\right\}, h_{i}^{j}$ denotes the first vertex of $B_{i}^{j}$.

Lemma 2.5. $\forall i \in\{1, \ldots, K-1\}, \quad \mathcal{C}\left(\varphi^{(i)}, G_{i}\right)-\mathcal{C}\left(\varphi^{(i-1)}, G_{i}\right) \leq 0$.

Proof. Clearly,

$$
\mathcal{C}\left(\varphi^{(i)}, G_{i}\right)-\mathcal{C}\left(\varphi^{(i-1)}, G_{i}\right)=\sum_{v \in V_{i}}\left(\mathcal{C}\left(\varphi^{(i)}, v_{i}\right)-\mathcal{C}\left(\varphi^{(i-1)}, v_{i}\right)\right)
$$

(a) $\mathcal{C}\left(\varphi^{(i-1)}, t_{i}\right)=\varphi^{(i-1)}(t)-\varphi^{(i-1)}\left(t_{i}\right) \geq 0$.

$\mathcal{C}\left(\varphi^{(i)}, t_{i}\right)=\varphi^{(i)}(t)-\varphi^{(i)}\left(t_{i}\right)=\sum_{l=i+1}^{K}\left|V_{l}\right|$ since vertices numbered between $\varphi^{(i)}\left(t_{i}\right)+1$ and $\varphi^{(i)}(t)-1$ in $\varphi^{(i)}$ are exactly those from $\bigcup_{l=i+1}^{K} V_{l}$.

Therefore, $\mathcal{C}\left(\varphi^{(i)}, t_{i}\right)-\mathcal{C}\left(\varphi^{(i-1)}, t_{i}\right) \leq \sum_{l=i+1}^{K}\left|V_{l}\right|$. 
(b) Let $\left(m_{\beta}\right),\left(u_{\beta}\right)$ and $\left(v_{\beta}\right)$ be the sequences previously defined, with $l=i$. We set $U=\left\{u_{\beta}, \beta \in\{1, \ldots, \alpha\}\right\}$. For every $\beta \in\{1, \ldots, \alpha-1\}$ and $q \in\{i+1, \ldots, K\}$, we also define $L_{\beta, q}$ as the set of indices $p \in\left\{1, \ldots, k_{q}\right\}$ of blocs $B_{q}^{p}$ numbered between $B_{i}^{m_{\beta}}$ and $B_{i}^{m_{\beta+1}}$ by $\varphi^{i-1}$. More formally,

$$
\begin{aligned}
& L_{\beta, q}=\left\{p \in\left\{1, \ldots, k_{q}\right\}, \varphi^{(i-1)}\left(h_{i}^{m_{\beta}}\right)<\varphi^{(i-1)}\left(h_{q}^{p}\right)<\varphi^{(i-1)}\left(h_{i}^{m_{\beta}+1}\right)\right\} \text { and } \\
& L_{\alpha, q}=\left\{p \in\left\{1, \ldots, k_{q}\right\}, \varphi^{(i-1)}\left(h_{i}^{m_{\alpha}}\right)<\varphi^{(i-1)}\left(h_{q}^{p}\right)\right\} .
\end{aligned}
$$

Then, for every $u_{\beta} \in U$, we have

$$
\mathcal{C}\left(\varphi^{(i)}, u_{\beta}\right)-\mathcal{C}\left(\varphi^{(i-1)}, u_{\beta}\right)=-\sum_{q=i+1}^{K} \sum_{p \in L_{\beta, q}}\left|B_{q}^{p}\right| .
$$

Therefore

$$
\sum_{u \in U} \mathcal{C}\left(\varphi^{(i)}, u\right)-\mathcal{C}\left(\varphi^{(i-1)}, u\right)=-\sum_{q=i+1}^{K} \sum_{\beta=1}^{\alpha} \sum_{p \in L_{\beta, q}}\left|B_{q}^{p}\right| .
$$

Now, for every $q \in\{i+1, \ldots, K\}$,

$$
\bigcup_{\beta=1}^{\alpha} L_{\beta, q}=\left\{p \in\left\{1, \ldots, k_{q}\right\}, \varphi^{(i-1)}\left(h_{i}^{1}\right)<\varphi^{(i-1)}\left(h_{q}^{p}\right)\right\} .
$$

Since all vertices from $V_{q}, q \in\{i+1, \ldots, K\}$ are numbered by $\varphi^{(i-1)}$ after $B_{i}^{1}$, we get $\bigcup_{\beta=1}^{\alpha} L_{\beta, q}=\left\{1, \ldots, k_{q}\right\}$ and

$$
\begin{aligned}
\sum_{u \in U} \mathcal{C}\left(\varphi^{(i)}, u\right)-\mathcal{C}\left(\varphi^{(i-1)}, u\right) & =-\sum_{q=i+1}^{K} \sum_{p=1}^{k_{q}}\left|B_{q}^{p}\right| \\
& =-\sum_{q=i+1}^{K}\left|V_{q}\right| .
\end{aligned}
$$

(c) Lastly, for every vertex $v \in V_{i}-\left\{t_{i}\right\}-U$ and for every $w \in \Gamma^{+}(v), \varphi^{(i)}(w)-$ $\varphi^{(i)}(v) \leq \varphi^{(i-1)}(w)-\varphi^{(i-1)}(v)$. Therefore

$$
\mathcal{C}\left(\varphi^{(i)}, v\right)-\mathcal{C}\left(\varphi^{(i-1)}, v\right) \leq 0 .
$$

Hence the result.

Lemma 2.6. For every $i \in\{1, \ldots, K-1\}$ and for every $l \in\{i+1, \ldots, K\}$, we have

$$
\mathcal{C}\left(\varphi^{(i-1)}, G_{l}\right)-\mathcal{C}\left(\varphi^{(i)}, G_{l}\right) \geq \sum_{j \in L_{l}}\left|B_{i}^{j}\right|
$$


where $L_{l}$ is the set of index $j \in\left\{2, \ldots, k_{i}\right\}$ such that $B_{i}^{j}$ is ordered in $\varphi^{(i-1)}$ after $B_{l}^{1}$, i.e.,

$$
L_{l}=\left\{j \in\left\{2, \ldots, k_{i}\right\}, \varphi^{(i-1)}\left(h_{l}^{1}\right)<\varphi^{(i-1)}\left(h_{i}^{j}\right)\right\} .
$$

Proof. Let $\left(m_{\beta}\right),\left(u_{\beta}\right)$ and $\left(v_{\beta}\right)$ be the sequences previously defined, for a fixed value $l \in\{i+1, \ldots, K\}$. We set $U=\left\{u_{\beta}, \beta \in\{1, \ldots, \alpha\}\right\}$. For every $\beta \in$ $\{1, \ldots, \alpha-1\}$, the set $L_{\beta}^{\prime}$ contains the indices $j \in\left\{1, \ldots, k_{i}\right\}$ of blocks $B_{i}^{j}$ ordered by $\varphi^{(i-1)}$ between $B_{l}^{m_{\beta}}$ and $B_{l}^{m_{\beta+1}}$. More formally,

$\forall \beta \in\{1, \ldots, \alpha-1\}$,

$$
\begin{aligned}
& \qquad L_{\beta}^{\prime}=\left\{j \in\left\{2, \ldots, k_{i}\right\}, \varphi^{(i-1)}\left(h_{l}^{m_{\beta}}\right)<\varphi^{(i-1)}\left(h_{i}^{j}\right)<\varphi^{(i-1)}\left(h_{l}^{m_{\beta+1}}\right)\right\} \\
& \text { and } L_{\alpha}^{\prime}=\left\{j \in\left\{2, \ldots, k_{i}\right\}, \varphi^{(i-1)}\left(h_{l}^{m_{\alpha}}\right)<\varphi^{(i-1)}\left(h_{i}^{j}\right)\right\} .
\end{aligned}
$$

(a) $\forall \beta \in\{1, \ldots, \alpha\}, \mathcal{C}\left(\varphi^{(i-1)}, u_{\beta}\right)-\mathcal{C}\left(\varphi^{(i)}, u_{\beta}\right)=\sum_{j \in L_{\beta}^{\prime}}\left|B_{i}^{j}\right|$.

Since $L_{l}=\bigcup_{\beta=1}^{\alpha} L_{\beta}^{\prime}$, we get

$$
\sum_{\beta=1}^{\alpha}\left(\mathcal{C}\left(\varphi^{(i-1)}, u_{\beta}\right)-\mathcal{C}\left(\varphi^{(i)}, u_{\beta}\right)\right)=\sum_{\beta=1}^{\alpha} \sum_{j \in L_{\beta}^{\prime}}\left|B_{i}^{j}\right|=\sum_{j \in L_{l}}\left|B_{i}^{j}\right| .
$$

(b) Lastly, for every vertex $v \in V_{l}-U$ and for every $w \in \Gamma^{+}(v), \varphi^{(i)}(w)-$ $\varphi^{(i)}(v) \leq \varphi^{(i-1)}(w)-\varphi^{(i-1)}(v)$. Therefore

$$
\mathcal{C}\left(\varphi^{(i)}, v\right)-\mathcal{C}\left(\varphi^{(i-1)}, v\right) \leq 0
$$

hence the result.

Lemma 2.7. For every $i \in\{1, \ldots, K-1\}$,

$$
\mathcal{C}\left(\varphi^{(i)}, G_{i+1}\right)-\mathcal{C}\left(\varphi^{(i-1)}, G_{i+1}\right) \leq \varphi^{(i-1)}\left(s_{i+1}\right)-\varphi^{(i)}\left(s_{i+1}\right)
$$

Proof. Vertices from $V_{i}$ are ordered consecutively by $\varphi^{(i)}$, so $\varphi^{(i)}\left(s_{i+1}\right)=\varphi^{(i)}\left(s_{i}\right)+$ $\left|V_{i}\right|$. Moreover,

$$
\varphi^{(i-1)}\left(s_{i+1}\right)=\varphi^{(i-1)}\left(s_{i}\right)+\left|B_{i}^{1}\right|=\varphi^{(i)}\left(s_{i}\right)+\left|B_{i}^{1}\right| .
$$

Therefore

$$
\varphi^{(i-1)}\left(s_{i+1}\right)-\varphi^{(i)}\left(s_{i+1}\right)=\left|B_{i}^{1}\right|-\left|V_{i}\right| .
$$

Moreover, $L_{i+1}=\left\{j \in\left\{2, \ldots, k_{i}\right\}, \varphi^{(i-1)}\left(h_{i+1}^{1}\right)<\varphi^{(i-1)}\left(h_{i}^{j}\right)\right\}=\left\{2, \ldots, k_{i}\right\}$.

Therefore, $\sum_{j \in L_{i+1}}\left|B_{i}^{j}\right|=\left|V_{i}\right|-\left|B_{i}^{1}\right|$, and we get the result by Lemma 2.6. 
Theorem 2.8 (Dominance of block orders). Let $G \in r$-2TSPG be the result of the parallel composition of $K$ graphs $G_{i} \in \operatorname{r-2TSPG}, \forall i \in\{1, \ldots, K\}$, and let $\varphi$ be an order of $G$. It is possible to build a block order $\varphi^{\prime}$ with no greater cost, i.e.,

$$
\mathcal{C}\left(\varphi^{\prime}, G\right) \leq \mathcal{C}(\varphi, G)
$$

Proof. In this section we have built a series of transformations of $\varphi=\varphi^{(0)}$ leading to a block order $\varphi^{(K-1)}$. Lemmas $2.2,2.3,2.5,2.7$ show that at each step $i \in$ $\{1, \ldots, K-1\}$, we have

$$
\begin{aligned}
\mathcal{C}\left(\varphi^{(i)}, G\right)-\mathcal{C}\left(\varphi^{(i-1)}, G\right)= & \mathcal{C}\left(\varphi^{(i)}, s\right)-\mathcal{C}\left(\varphi^{(i-1)}, s\right)+\mathcal{C}\left(\varphi^{(i)}, G_{i}\right)-\mathcal{C}\left(\varphi^{(i-1)}, G_{i}\right) \\
& +\mathcal{C}\left(\varphi^{(i)}, G_{i+1}\right)-\mathcal{C}\left(\varphi^{(i-1)}, G_{i+1}\right) \\
& +\sum_{l=i+2}^{k} \mathcal{C}\left(\varphi^{(i)}, G_{l}\right)-\mathcal{C}\left(\varphi^{(i-1)}, G_{l}\right) \\
\leq & 0 .
\end{aligned}
$$

Therefore, $\mathcal{C}\left(\varphi^{(K-1)}, G\right) \leq \mathcal{C}\left(\varphi^{(0)}, G\right)$, hence the result.

\section{Optimal BLOCK ORDER}

The aim of this section is to characterize an optimal block order. An $\mathcal{O}\left(|V|^{2}\right)$ time complexity algorithm is then derived. Let us first evaluate the cost of a block order for a parallel composition:

Lemma 3.1. Let $G=(V, A)$ the parallel composition of $K$ graphs $G_{i}=\left(V_{i}, A_{i}\right), i \in$ $\{1, \ldots, K\}$ and let $\varphi$ be a block order such that, for any couple of integers $(i, j) \in$ $\{1, \ldots, K\}^{2}$ with $i<j, \forall x \in V_{i}, \forall y \in V_{j}, \varphi(i)<\varphi(j)$. Then,

$$
\mathcal{C}(\varphi, G)=(K+1)+\sum_{i=1}^{K} \mathcal{C}\left(\varphi, V_{i} \backslash\left\{t_{i}\right\}\right)+\sum_{i=1}^{K-1} i\left|V_{i}\right|+(K-1)\left|V_{K}\right| .
$$

Proof. $\mathcal{C}(\varphi, G)$ can be decomposed into three terms:

$$
\mathcal{C}(\varphi, G)=\mathcal{C}(\varphi, s)+\sum_{i=1}^{K} \mathcal{C}\left(\varphi, V_{i} \backslash\left\{t_{i}\right\}\right)+\sum_{i=1}^{K} \mathcal{C}\left(\varphi,\left\{t_{i}\right\}\right) .
$$

(1) $\mathcal{C}(\varphi, s)=\varphi\left(s_{K}\right)-\varphi(s)=1+\sum_{i=1}^{K-1}\left|V_{i}\right|$

(2) For any $i \in\{1, \ldots, K\}, \mathcal{C}\left(\varphi,\left\{t_{i}\right\}\right)=\varphi(t)-\varphi\left(t_{i}\right)=1+\sum_{j=i+1}^{K}\left|V_{j}\right|$. So,

$$
\sum_{i=1}^{K} \mathcal{C}\left(\varphi,\left\{t_{i}\right\}\right)=K+\sum_{i=1}^{K}(i-1)\left|V_{i}\right|
$$


Adding these two terms, we get

$$
\mathcal{C}(\varphi, s)+\sum_{i=1}^{K} \mathcal{C}\left(\varphi,\left\{t_{i}\right\}\right)=(K+1)+\sum_{i=1}^{K-1} i\left|V_{i}\right|+(K-1)\left|V_{K}\right|
$$

hence the lemma.

Theorem 3.2. Let $G=(V, A)$ be the parallel composition of $K$ graphs $G_{1}, \ldots, G_{K}$ such that $\left|V_{1}\right| \geq \ldots \geq\left|V_{K-2}\right|$ and $\left|V_{K-2}\right| \geq \max \left(\left|V_{K-1}\right|,\left|V_{K}\right|\right)$. Let $\varphi^{\star}$ be a block order such that

(1) $\forall i \in\{1, \ldots, K\}, \mathcal{C}\left(\varphi^{\star}, V_{i} \backslash\left\{t_{i}\right\}\right)$ is minimum;

(2) for every couple of integers $(i, j) \in\{1, \ldots, K\}^{2}$ with $i<j, \forall(x, y) \in$ $V_{i} \times V_{j}, \varphi^{\star}(x)<\varphi^{\star}(y)$.

Then, $\varphi^{\star}$ is optimal.

Proof. From Theorem 2.8, block orders are dominant. $\varphi^{\star}$ also minimizes the cost function $\mathcal{C}(\varphi, G)$ expressed by Lemma 3.1 thus the result.

Theorem 3.3. Let $G=(V, A)$ be a r-2TSPG graph. An optimal order for minDSC can be computed polynomially with a time complexity bounded by $\mathcal{O}\left(|V|^{2}\right)$.

Proof. A simple algorithm to compute an optimal block order can be derived from Theorem 3.2. Assuming that an optimal order was previously computed for subgraphs $G_{1}, \ldots, G_{K}$, the complexity for parallel composition is in $\mathcal{O}(K \log K)$.

Let us now denote $u_{n}$ the complexity of the computation of an optimal order for a graph with $n$ vertices. We prove that $u_{n} \in \mathcal{O}\left(n^{2}\right)$ by recurrence. Let $G=(V, A)$ be a series or parallel composition of subgraphs $G_{1}, \ldots, G_{K}$. Then, $n=n_{1}+\ldots+n_{K}+2$, with $n_{i}=\left|V_{i}\right|$ for $i \in\left\{1, \ldots, n_{K}\right\}$. Then, there exists a constant $M>0$ such that

$$
u_{n} \leq u_{n_{1}}+\ldots+u_{n_{K}}+M K \log K
$$

Setting $M^{\star}=\max \left(u_{2}, M\right)$, we prove by recurrence that $\forall n \geq 2, u_{n} \leq M^{\star} n^{2}$.

Let us assume that $u_{n_{j}} \leq M^{\star} n_{j}^{2}, \forall j \in\{1, \ldots, K\}$. Then,

$$
u_{n} \leq M^{\star}\left(n_{1}^{2}+\ldots+n_{K}^{2}+K \log K\right) .
$$

Now, since $n_{j} \geq 2, \forall j \in\{1, \ldots, K\}$,

$$
2 \sum_{i=1}^{K-1} n_{i} \sum_{j=i+1}^{K} n_{j} \geq 8 \sum_{i=1}^{K-1}(K-i)=4 K(K-1) .
$$

As $4 K(K-1) \geq K \log K$

$$
2 \sum_{i=1}^{K-1} n_{i} \sum_{j=i+1}^{K} n_{j} \geq K \log K \text { and }
$$




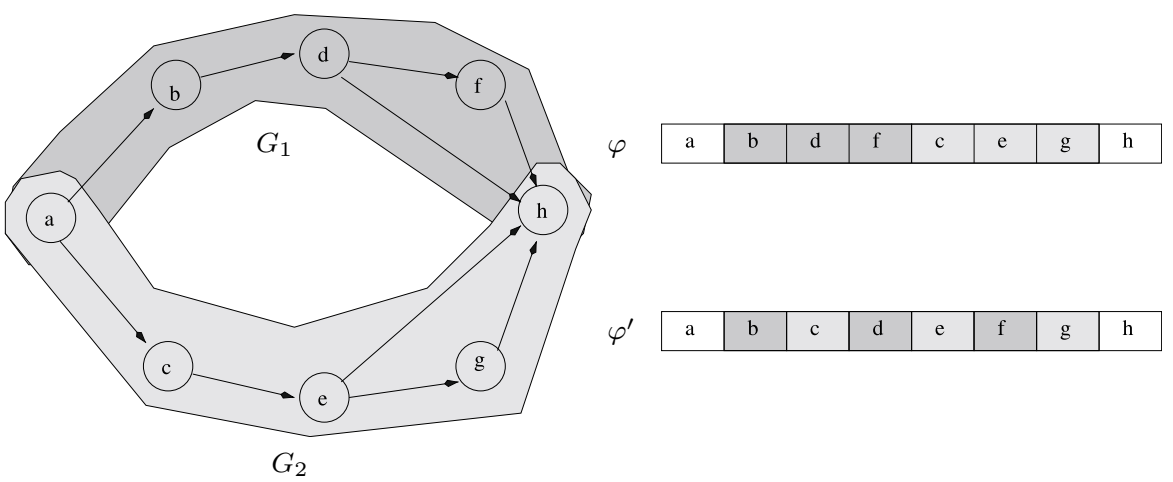

FiguRE 5. $\mathcal{C}(\varphi)=18>\mathcal{C}\left(\varphi^{\prime}\right)=17$

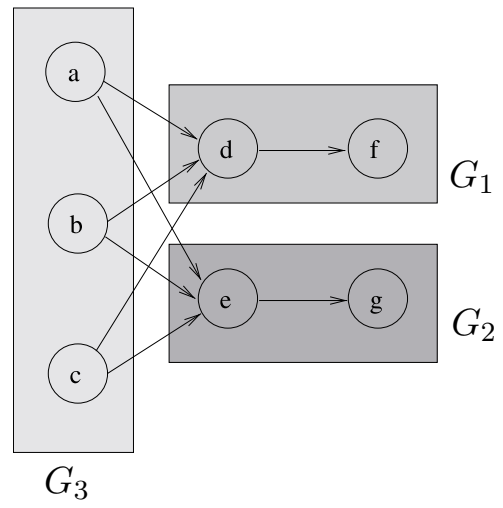

$\varphi$

\begin{tabular}{|l|l|l|l|l|l|l|}
\hline a & b & c & d & f & e & g \\
\hline
\end{tabular}

Figure $6 . \mathcal{C}(\varphi)=14>\mathcal{C}\left(\varphi^{\prime}\right)=13$

$$
u_{n} \leq M^{\star}\left(n_{1}^{2}+\ldots+n_{K}^{2}+2 \sum_{i=1}^{K-1} n_{i} \sum_{j=i+1}^{K} n_{j}\right)=M^{\star} n^{2},
$$

which completes the proof.

\section{Conclusion}

Our work leads to another polynomial class for minDSC. In an attempt to loosen the algorithm hypothesis, we tried to apply the block method to the class of 2-terminal graphs. This study leads to a counter example, as shown in Figure 5. We also tried to apply the block method in order to find a polynomial algorithm for the class SP. A counter example is shown in Figure 6. These examples show that Theorem 2.8 cannot be extended to 2TSPG nor SP. One prospect of our work is therefore to study the complexity of minDSC for graphs belonging to such classes. 


\section{REFERENCES}

[1] T. Bossart, A. Munier and F. Sourd, Two models for the optimization of integrated circuit simulators. Discrete Appl. Math. 155 (2007) 1795-1811.

[2] T. Bossart, Optimisation de la mémoire cache pour la simulation de circuits. Ph.D. thesis, Université Pierre et Marie Curie (2006).

[3] P. Brucker, Scheduling Algorithms. Springer-Verlag New York, Inc., Secaucus, NJ, USA (1995).

[4] P. Chrétienne and C. Picouleau, Scheduling with communication delays: a survey, in Scheduling Theory and its Applications, edited by P. Chretienne, E.G. Jr Coffman, J.K. Lenstra and Z. Liu, Chap. 4. John Wiley \& Sons (1995) 65-90.

[5] L.A.M. Schoenmakers, A new algorithm for the recognition of series parallel graphs, Technical Report CS-R9504 Centrum voor Wiskunde en Informatica (1995).

[6] R. Sethi, Complete register allocation problems. SIAM J. Computing 4 (1975) 226-248.

[7] W.E. Smith, Various optimizers for single stage production. Naval Research Logistics Quarterly 3 (1956) 59-66.

[8] J. Valdes, R.E. Tarjan and E.L. Lawler, The recognition of series parallel digraphs, in Proceedings of the eleventh annual ACM symposium on Theory of computing. ACM Press (1979) $1-12$. 